Proceedings of the Pakistan Academy of Sciences:

A: Physical and Computational Sciences 58(3): 67-77 (2021)

Copyright (C) Pakistan Academy of Sciences

ISSN (Print): 2518-4245; ISSN (Online): 2518-4253

https://doi.org/10.53560/PPASA(58-3)752

\title{
Multiple Input Single Output DC to DC Converter Control Using Kalman Filter for Microgrid Applications
}

\author{
Waqas Farooq ${ }^{1}$, Muhammad J. Alvi", and Tahir Izhar ${ }^{1}$ \\ ${ }^{1}$ Department of Electrical Engineering, University of Engineering and Technology, \\ Lahore, Pakistan \\ ${ }^{2}$ Department of Electrical Engineering, NFC Institute of Engineering and Fertilizer Research, \\ Faisalabad, Pakistan
}

\begin{abstract}
Renewable energy system (RES) based microgrid applications have grown extensively over the recent years. Owing to power fluctuations in RES, acquiring stable and accurate output voltage at the DC Bus is a major concern in DC microgrid applications. Presently, switching, as well as, prediction of output voltage for RES is quite slow and total harmonic distortion cannot be reduced to a minimal level. Accordingly, this research developed a controller for Multiple Input Single Output (MISO) DC to DC converter and a Kalman filter. Initially, four seriesconnected PV panels were modelled and analysed. A boost converter was used to combine PV panels' output and provide a single output at the DC Bus. Perturb and Observe, a Maximum Power Point Tracking (MPPT) algorithm, was used to retrieve optimal power from modelled RES. Analysis revealed that the output voltage waveform contained harmonics and had a Total Harmonic Distortion (THD) of $27.73 \%$. Thus, a Kalman filter was modeled and analysed to remove the harmonics. The THD value was consequently reduced to $2.1 \%$, which is quite within the allowable limit prescribed by IEEE, for the THD of a PV system. Analysis revealed that a stable and accurate output from a PV based RES could be achieved with the proposed scheme, and further THD was also well within limits prescribed by IEEE
\end{abstract}

Keywords: Converter, Hybrid Energy Systems, Kalman Filter, Microgrid, Maximum Power Point Tracking, Photo Voltaic, Renewable Energy, Total Harmonics Distortion.

\section{INTRODUCTION}

Proliferation in demand for electrical load and the requirement for stability and reliability has resulted in an extensive advancement in the development of microgrid systems. In a microgrid system, multiple wind energy (WE) and photovoltaic (PV) systems are integrated to fulfil the electrical load demand at micro levels [1]. This technique facilitates the minimisation of load bulk over the main power system as well as eliminates the requirement of implementation of the power transmission system. However, due to possible variation in the wind speed and sunlight, the output of renewable energy systems (RES) may vary on an intermittent basis [1]. For optimal energy, a combination of PV system and WE system is preferred and multi-input converters facilitate the amulgation of multiple energy sources while providing at least one output $[2,3]$. Different topologies for multi-input DC-DC converters are discussed in the literature. Mirzapour et al. [4], presents a high voltage-conversion ratio integrated multiple-input buck converter and describe that the load can be supplied separately or concurrently by multiple DC input sources. Prasad et al. [5], discusses some basic topologies which could be paired with sepic, buck-boost, Zeta, boost and Cuk converters. When joned with traditional buck converter, these schemes result in a significant decrease in the output voltage. However, in case of these techniques, the prediction of output voltage and switching is not fast enough, and THD cannot be reduced to a negligible level. Single-tuned Passive Filter can reduce THD of the system to fulfil the IEEE 519-1992 standard requirements but is still not fast enough to predict the future voltage

Received: July 2021; Accepted: September 2021

*Corresponding Author: Muhammad Junaid Alvi <junaidalvi@iefr.edu.pk> 
variation with minimum error. Multi-input DCDC converters allow energy from one or multiple sources to be shared with the load. Control signals of multiple converters should be synced because they are not self-contained [6- 10]. Following the studies on controllers for DC-DC converters, extensive research on Kalman filter with power electronic converters emerges [11-14]. The Kalman filter can simulate and analyse the data of a learning algorithm-generated implication system. Kalman filter governs the power dispersal from every energy resource of a multiple-input converter $[15,16]$. Kalman filter is faster and predicts more accurately than Adaptive Neuro-Fuzzy implication System [17, 18].

In this study, a controller for Multiple Input Single Output (MISO) DC to DC converter along with a Kalman filter was developed to facilitate the integration of multiple RES in order to provide a single output at the DC Bus. Accordingly, four series-connected PV panels were initially modelled and analysed to get a stable and accurate output voltage regardless of the possible supply variations from a PV-based RES. Boost converter combined the output of two PV panels and in order to retrieve optimal power, Perturb and Observe (P\&O), an MPPT algorithm, was used. Moreover, two outputs of four PV panels were combined using another boost converter to get a single DC output from all four PV panels. Analysis revealed that the output voltage waveform contained harmonics having a THD of $27.73 \%$. Thus, the Kalman filter was modelled and analysed to remove the harmonics and resultantly the THD value was reduced to $2.1 \%$. The Kalman filter was used in the close loop feedback mechanism in such a way that the output generated was regulated instantly. Analysis revealed that a stable, accurate and nearly sine wave for a PV based RES could be achieved with the proposed scheme and further THD was also well within the limits as prescribed by IEEE for a PV system.

\subsection{Kalman Filter}

Kalman filter effectively delineates the probabilistic structure of experimental measurements [19,2022]. It is an effective tool to minimise the distortion in the output of a converter. It estimates the states on the basis of a linear dynamic system in a state space format. The process model expresses the development of a state from $\mathrm{k}-1$ to $\mathrm{k}$, as is shown in equation (1) [23].

$$
\mathrm{x}_{\mathrm{k}}=\mathrm{Fx}_{\mathrm{k}-1}+\mathrm{bu}_{\mathrm{k}-1}+\mathrm{w}_{\mathrm{k}-1}
$$

In equation (1), $\mathrm{F}$ represents the state transition matrix, $\mathrm{w}_{\mathrm{k}-1}$ represents process noise vector, and $\mathrm{b}$ represents control-input matrix,. Equation (2) shows that the process model and measurement model are paired together, where as, measurement model illustrates the correlation between measurement and state at a time step $\mathrm{k}$ [23].

$$
\mathrm{Z}_{\mathrm{k}}=\mathrm{Hx}_{\mathrm{k}}+\mathrm{v}_{\mathrm{k}}
$$

In equation (2), $Z_{k}$ represents measurement vector, $v_{k}$ represents measurement noise vector, and $\mathrm{H}$ represents measurement matrix. Provided an initial estimate of $x_{k}, Z_{1}$ to $Z_{k}$, as well as, $B, R, F$, $\mathrm{Q}$ and $\mathrm{H}$, Kalman filter approximates $\mathrm{x}_{\mathrm{k}}$ at a time $\mathrm{k}$ [23]. Figure 1 shows the functionality of Kalman

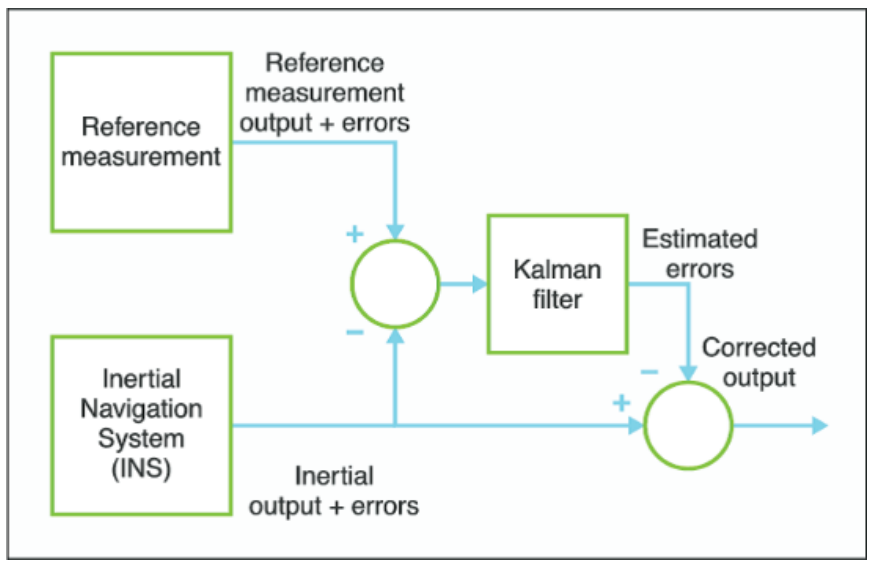

Fig. 1. Schematic of Kalman filter . 
filter.

\section{MATERIALS AND METHODS}

This research wants DC output having the lowest THD regardless of the input variations. We used Kalman Filter as it is fast and predicts future voltage variations values with accuracy. Accordingly, four PV panels were initially modelled and analysed to get a stable and accurate output voltage regardless of the possible supply variations from a PV-based RES. The PV block, used in the modeling is basically a five-parameter model using a light-generated current source $\left(I_{L}\right)$, diode, series resistance $\left(R_{s}\right)$, and shunt resistance $\left(\mathrm{R}_{\mathrm{sh}}\right)$ to represent the irradiance and temperature-dependent $\mathrm{I}-\mathrm{V}$ characteristics of the modules. The output of two PV panels was combined using a boost converter, and in order to extract maximum output from $\mathrm{PV}$ panels, $(\mathrm{P} \& \mathrm{O})$, an MPPT algorithm was used. This algorithm adjusts the duty cycle for switching the MOSFET of the boost converter. The other two solar panels were combined using the same technique as explained earlier. Furthermore, to have maximum power, as well as, to have a single output voltage from all PV panels, the two outputs from four PV panels were again integrated by using a boost converter. After performing power quality analysis, it was revealed that the developed model had a very high THD value, and it does not meet the THD limits for a PV system as specified by IEEE. Thus, in order to minimise the harmonics, the Kalman filter was modelled and analysed. Resultantly, the THD value of the system was observed to be well within limits for THD in PV systems, as specified by IEEE. The Kalman filter was used in the close-loop feedback mechanism in such a way that the output generated was regulated instantly. Figure 2 represents the complete schematic of the proposed scheme.

PV panels provide fluctuating power, and it depends upon shadow, solar irradiation and temperature. Thus, a MPPT algorithm is essentially required to get optimal power from PV panels. In this research, $\mathrm{P} \& \mathrm{O}$ was implemented for retrieving optimal power from PV panels. Figure 3 shows the flowchart of P\&O algorithm for MPPT of PV panels. Accordingly, perturbation was performed for the power and voltage of PV module. Output power was periodically measured and was compared with the preceding power. In case of an increase in output power, same procedure was repeated otherwise perturbation was reversed. Further, PV module voltage was decreasd, as well as, increased and the power profile of PV module was observed along with respectively. In case, increased voltage lead to increased power, it revealed that the operating point of the PV module was on the left of maximum power point. Accordingly, perturbation was performed towards the right in order to get to the maximum power point. Alternatively, when voltage rise led to decreased power, it revealed that the operating point of PV module was on the right of maximum power point. Resultantly, perturbation was performed to the left in order to get to a maximum power point. In order to measure the voltage of the battery and PV module, an MPPT charge controller was attached between the battery and PV module. The microcontroller then calculated and compared the existing power Pnew with the previous power Pold. In case, Pnew was greater than the Pold, duty cycle of PWM was increased. In case, If Pnew was observed to be lesser than Pold, duty cycle of PWM was reduced.

The Kalman filter algorithm comprises two stages: prediction and update (alternatively, propagation and correction). Moreover, an initialisation stage is essentially required. Accordingly, the initial estimate of error covariance matrix, $\mathrm{P}^{+}$, and state estimate, $\hat{\mathrm{x}} 0^{+}$, is mandatory. (Table 1) and (Table 2) summarises the Kalman filter algorithm for prediction and update respectively, used in this research.

In (Table 1), $\hat{x}$ represents an approximation of $x$. Further, $(+)$ and $(-)$ represent updated and predicted estimates, respectively, whereas $\mathrm{P}$ is the state error covariance. Further, $\operatorname{cov}(\mathrm{x})=\mathrm{E}[(\mathrm{x}-\hat{\mathrm{x}})(\mathrm{x}-\hat{\mathrm{x}}) \mathrm{T}] \mathrm{T}$ describes the covariance of a random variable $\mathrm{x}$.

(Table 2) represents update stage, and measurement residual was initially computed in this stage. Measurement residual is the difference of true and estimated measurement, zk and Hx̂k-, respectively. Initially, the filter estimates the current measurement, and then, residual is multiplied with Kalman gain to determine the correction in the estimate. Finally, the Kalman filter calculated the updated error covariance, $\mathrm{Pk}^{+}$, which was later on used in following time step. 


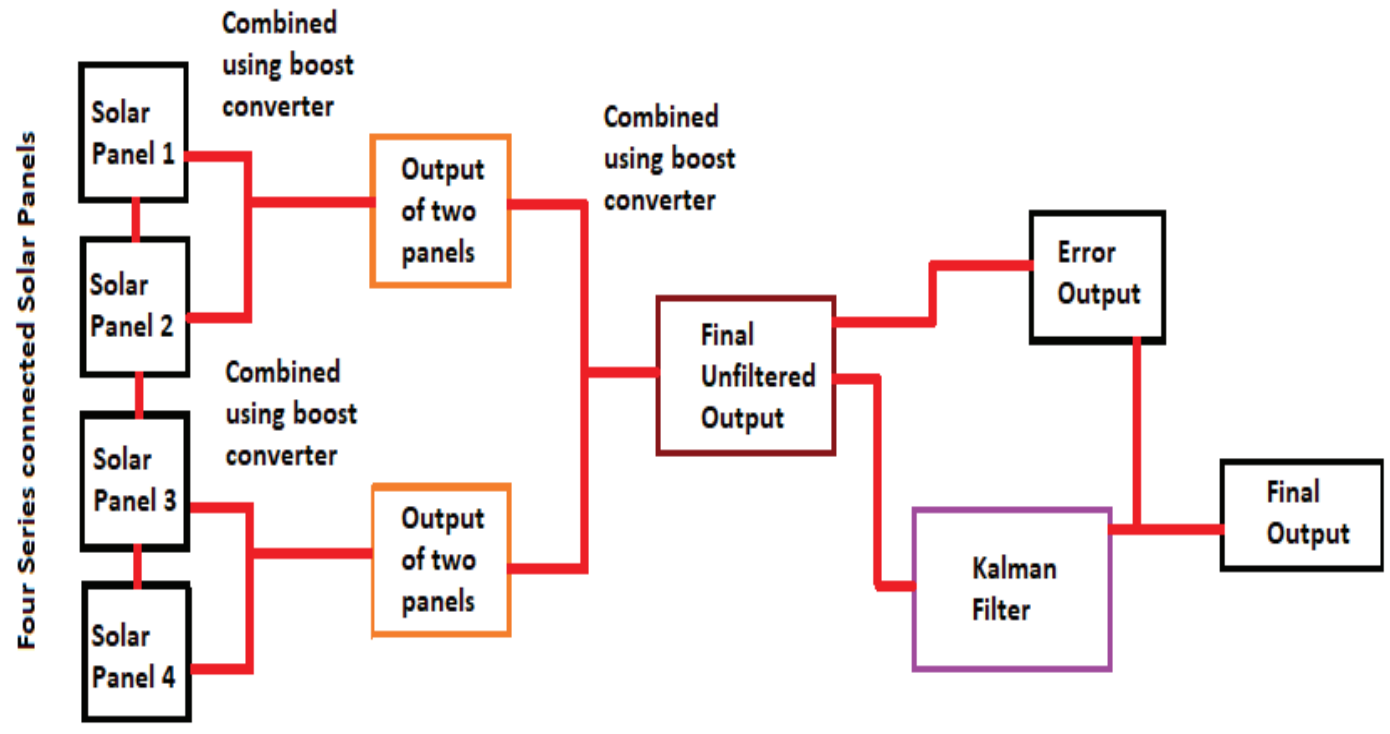

Fig. 2. Proposed schematic for the control of MISO DC to DC converter by using a Kalman filter for DC Microgrid applications.

Table 1. Kalman filter prediction model.

$\begin{array}{cc}\text { Predicated state estimation } & \boldsymbol{x}_{\boldsymbol{k}}^{\wedge}=\mathbf{F} \boldsymbol{x}_{k-1}^{+}+\mathbf{B} \boldsymbol{u}_{k-1} \\ \text { Predicted error covariance } & \boldsymbol{P}_{\boldsymbol{k}}^{-}=\boldsymbol{F} \boldsymbol{P}_{k-1}^{+} \boldsymbol{F}^{\boldsymbol{T}}+\boldsymbol{Q}\end{array}$

Table 2. Kalman filter updated model.

Measurement residual

Kalman gain

Updated state estimate

Updated error covariance

$$
\begin{gathered}
y \sim_{k}=z_{k}-H x_{k}^{-} \\
K_{k}=P_{k}^{-} H^{T}\left(R+H P_{k}^{-} H^{T}\right)^{-1} \\
x_{k}^{+}=x_{k}^{\wedge}+K_{k} y^{\sim} \\
P_{k}^{+}=\left(I-K_{k} H\right) P_{k}^{-}
\end{gathered}
$$

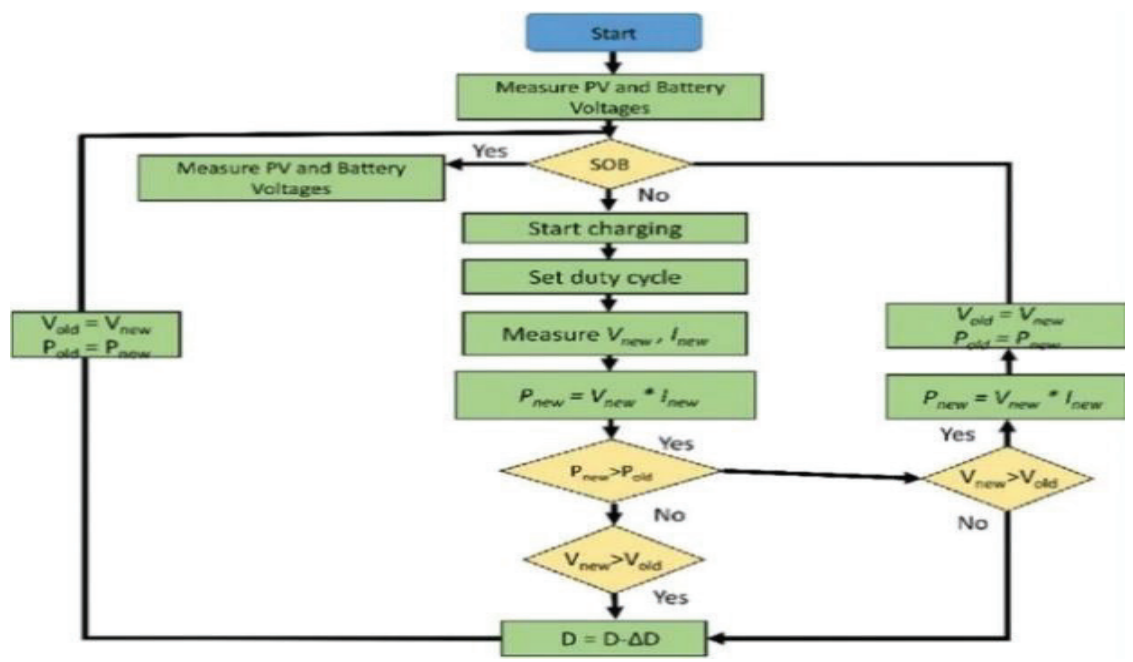

Fig. 3. Flowchart of perturb and observe (P\&O) algorithm for MPPT of PV panels. 


\section{RESULTS AND DISCUSSION}

As mentioned in section 2, four series connected PV panels were used in the modelling and analysis. So, to have an in-depth understanding of the system's response, investigations were initially conducted regarding the current, voltage and power profiles of one single PV panel. The PV panel model used in the modelling and analysis had a settling time of 0.3 sec. However, initially, for a duration of up to 0.1 $\mathrm{sec}$, voltage, current and power fluctuations were observed to be quite high, whereas, the respective fluctuations reduced gradually in the next $0.2 \mathrm{sec}$. Finally, after the settling time $(0.3 \mathrm{sec})$ passes, the voltage, current and power fluctuations stabilised. Higher voltage fluctuations were observed because the solar panel is a non linear device. The voltage, current and power waveforms of one single PV panel are shown in Figure 4, Figure 5 and Figure 6 , respectively, having time along the $\mathrm{x}$-axis and voltage, current and power along the y-axis, respectively.

Boost converterwasused in the developed model to extract maximum power from the developed PV model. PV panel provides fluctuating power, due to which, it cannot be directly utilised as a voltage input to the MISO converter, and consequently, a boost converter is essentially required. Boost converter provides maximum powers while operating in both cycles of the switching operation. Figure 7, Figure 8 and Figure 9 represent output voltage, current and power waveforms of a boost converter, respectively, having time on the $\mathrm{x}$-axis and voltage, current and power on the y-axis, respectively. A critical comparison between Figure 4, Figure 5, Figure 6 and Figure 7, Figure 8, Figure 9 respectively reveals that voltage, current and power, in the later, stabilises more quickly. Additionally, the voltage in Figure 7 also takes lesser time for achieving the constant DC value.

The switching of the boost converter was made basically for the duty cycle adjustment. The pulses provided the boost converter's turn on and turn off mechanism. In this way, maximum power was collected at the output of the converter. The pulse waveform for the boost converter is shown in Figure 10, and the pulse waveform for the main converter is shown in Figure 11.
MISO converter was used for obtaining a single output from multiple inputs. Thus, the output voltage of the MISO converter comprises the sum of voltages of all individual sources. Figure 12 represents the output voltage of the MISO converter. Further, the output current of a MISO converter is the same as the current of multiple input sources. Accordingly, Figure 13 represents the output current of a MISO converter and is of the same magnitude as compared to the currents shown in Figure 5 and Figure 8. Moreover, the power output of the MISO converter was determined with the help of voltage and current measured for the MISO converter and is shown in Figure 14. This waveform was inverted to perform the FFT analysis as we actually obtained DC output form MISO converter and FFT cannot be performed on a DC waveform. Consequently, we obtained 27\% THD in the final output waveform without Kalman Filtration.

As expressed in section 3, the voltage waveform of the MISO converter, presented in Figure 12, contained many harmonics and had a THD of $27.73 \%$, so harmonic suppression was essentially required. Thus, these harmonics were reduced with the help of a Kalman filter. This filtration process involved the creation of a measured signal of MISO converter, which yielded the estimated value of the voltage. The error was calculated by subtracting the estimated signal from the actual signal. Figure 15 compares the actual voltage signal of a MISO converter, subjected to the load, with the signal after Kalman Filter based filtration was implemented. The Kalman filter was used in a close loop feedback mechanism in such a way that output generated was regulated instantly. The estimated signal of the voltage was also created by utilising the Kalman filter and is presented in the Figure 16. This is the actual signal of the converter that is yielded as the output waveform. This voltage is also fed to the inverter for inversion of DC to AC. The voltage waveform generated by the inverter has almost sine wave and a THD of $2.10 \%$, which is quite lesser as compared to the allowable limit of $5 \%$, as prescribed by IEEE, for the THD of a PV system.

Figure 17 presents the harmonics in the final output waveform. The DC output obtained after Kalman filtration is inverted, which results in a nearly sinusoidal waveform. The FFT analysis of 


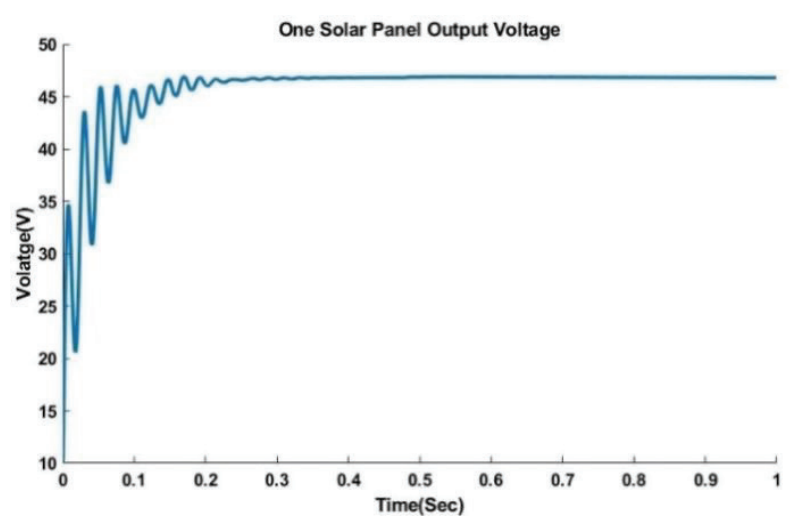

Fig. 4. Output voltage of a single PV panel.

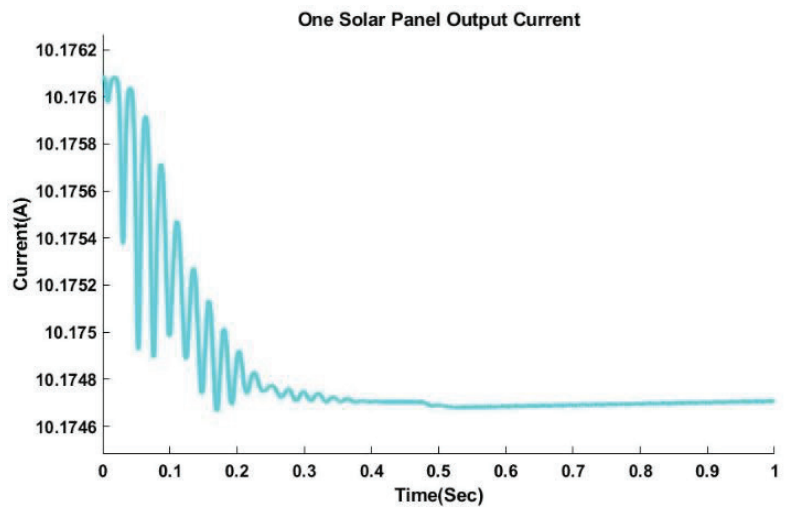

Fig. 5. Output current of a single PV panel.

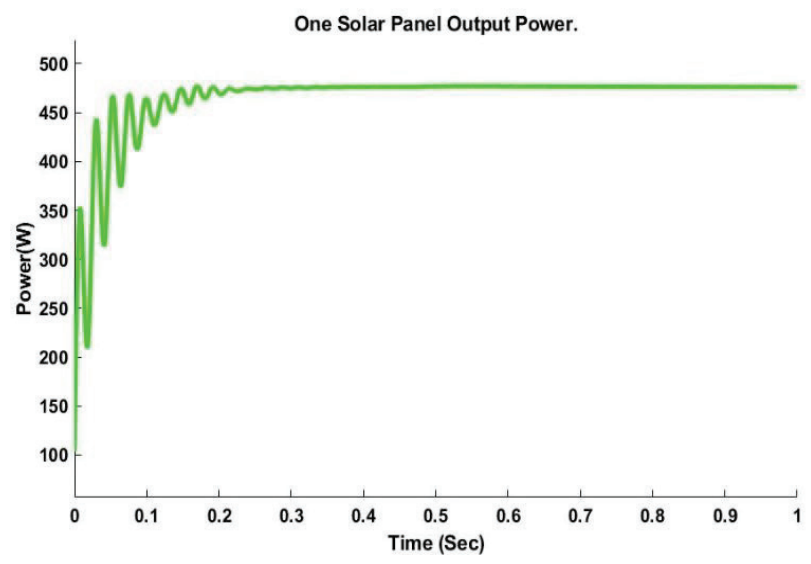

Fig. 6. Output power of a single PV panel.

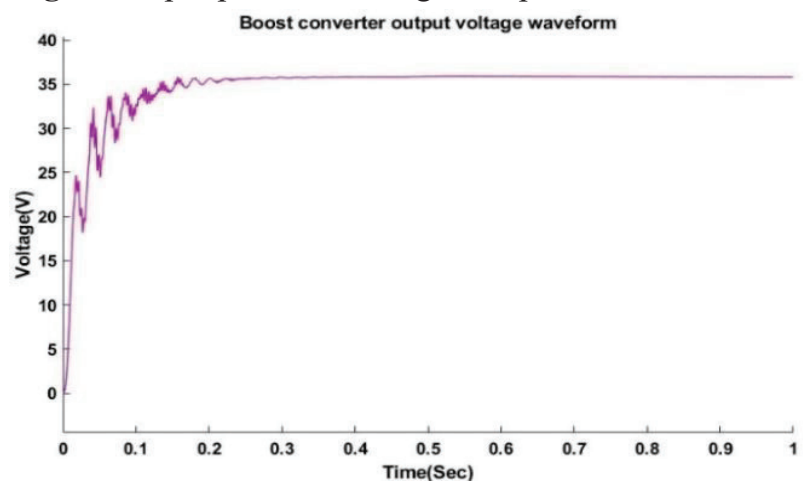

Fig. 7. Output voltage of a boost converter. 


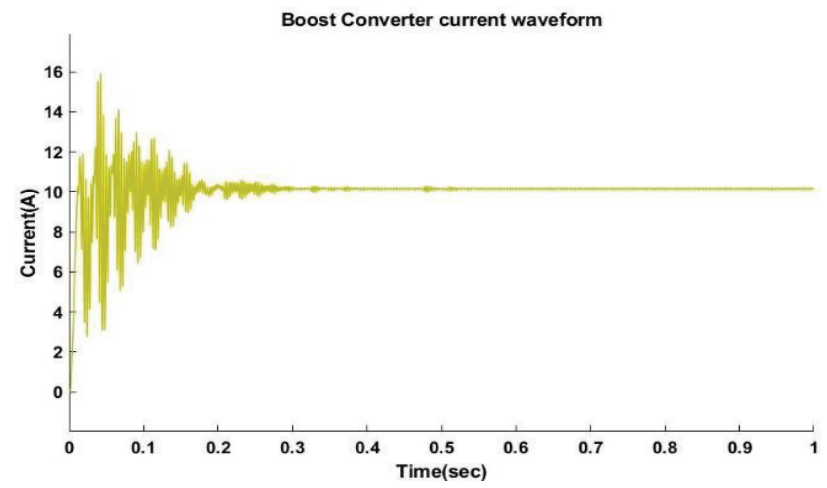

Fig. 8. Output current of a boost converter.

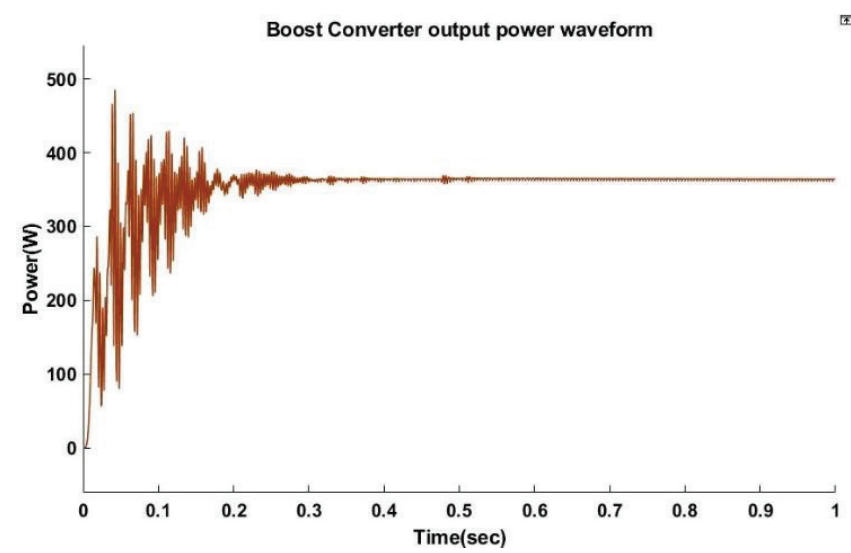

Fig. 9. Output power of a boost converter

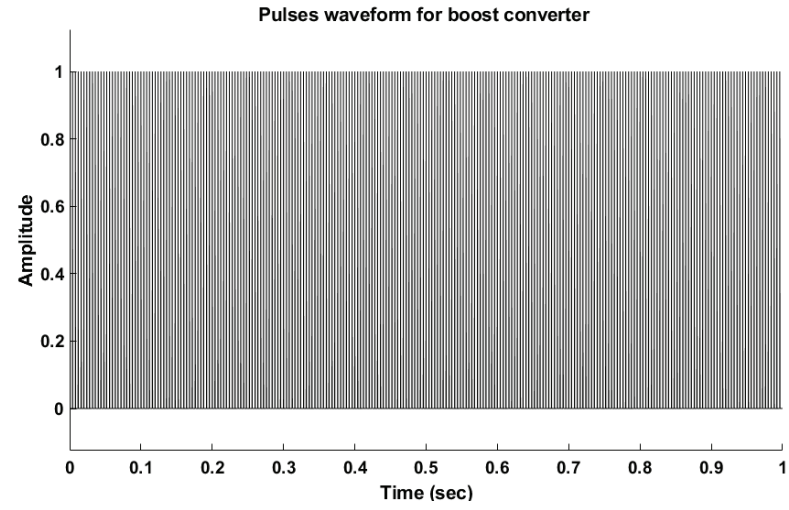

Fig. 10. Pulse waveform for boost converter. 


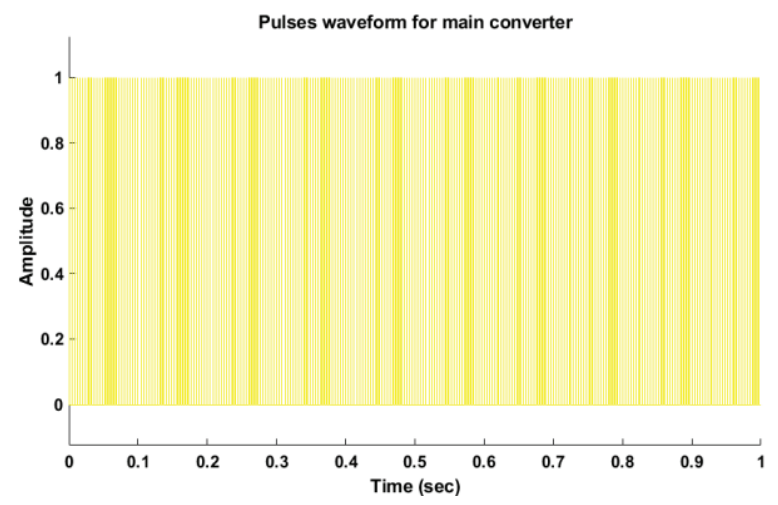

Fig. 11. Pulse waveform for main converter.

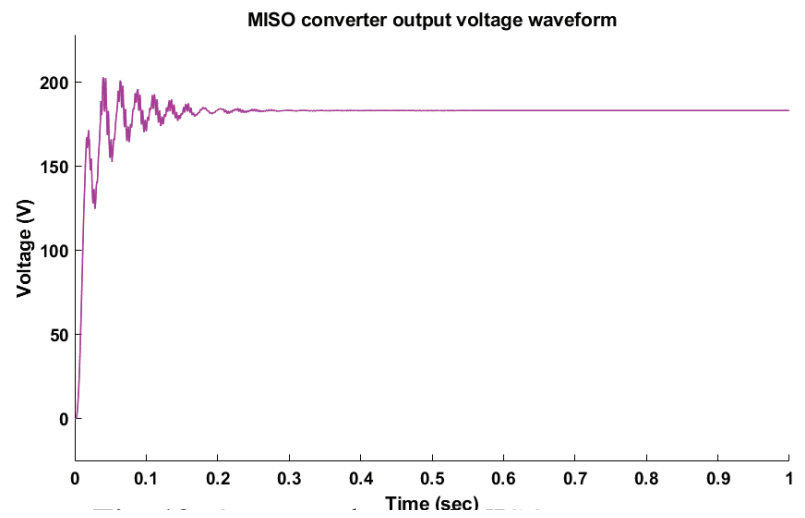

Fig. 12. Output voltage of MISO converter.

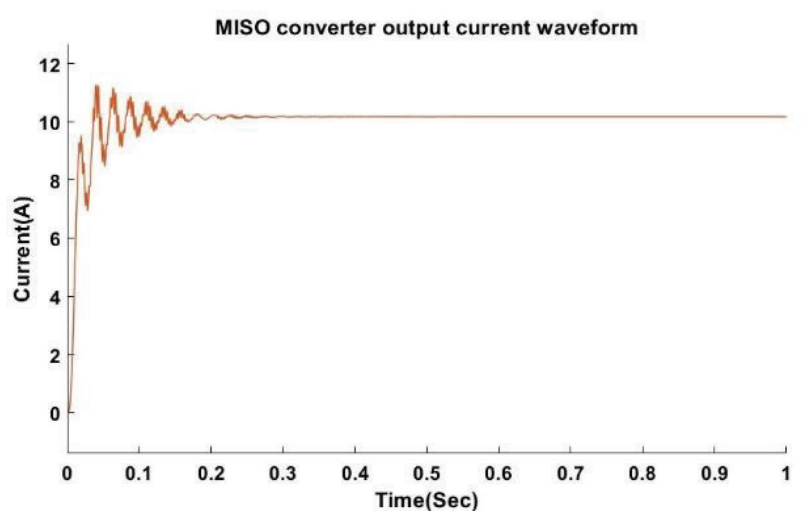

Fig. 13. Output current of MISO converter.

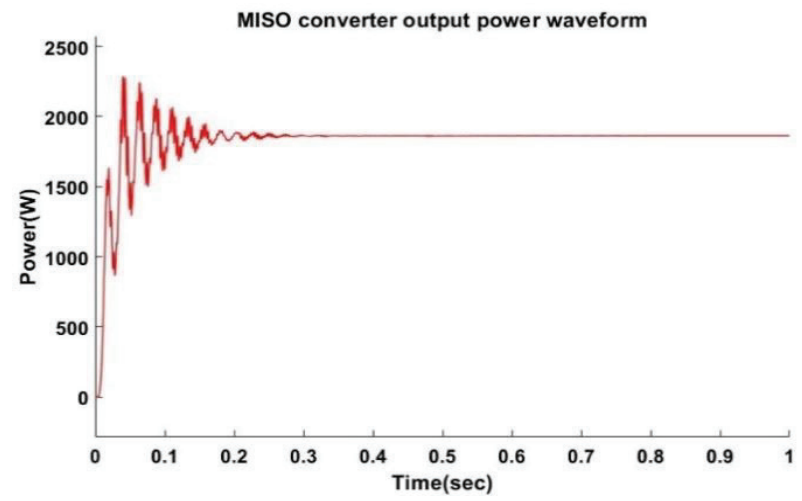

Fig. 14. MISO converter output power waveform 


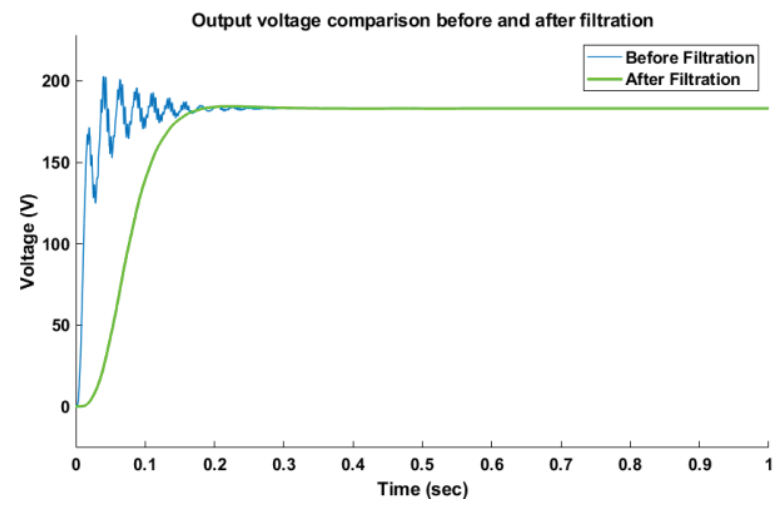

Fig. 15. Comparison between the output voltage of a MISO converter, before and after filtration.

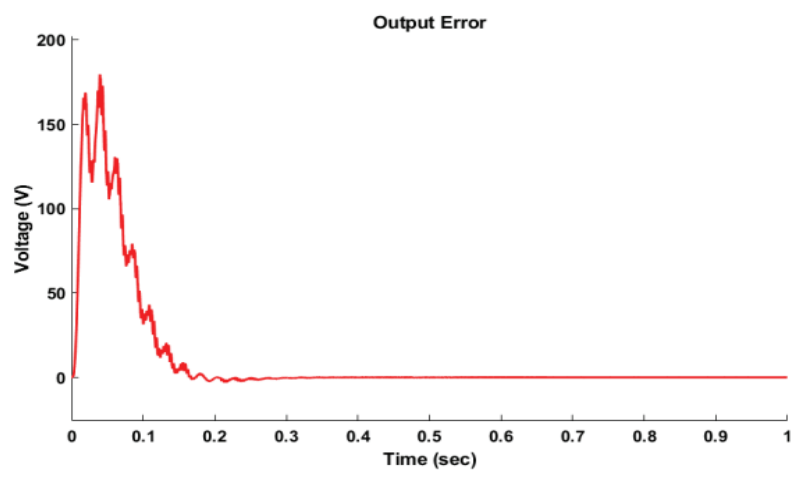

Fig. 16. Error estimation in the output voltage
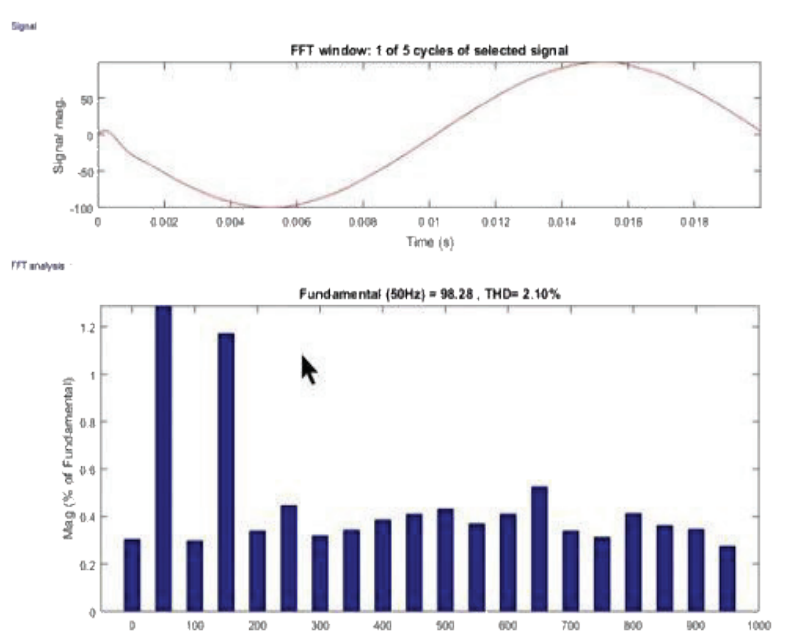

Frequency $(\mathrm{Hz})$

Fig. 17. THD of final output waveform. 
resulted waveform showed that the waveform had a THD value of $2.10 \%$, which is well within the allowable limits for a PV system.

\section{CONCLUSIONS}

In microgrid applications, fast prediction of output voltage and harmonic reduction are required. Existing techniques, such as a single-tuned passive filter, can reduce the THD up to $4.77 \%$, but still, it cannot predict the future voltage variation with minimum error. Thus, to get steady output voltage along with a lesser THD, a controller for MISO DC to DC converter and a Kalman filter was developed in this research. The PV model, used in the modelling and analysis was observed to have a settling time of $0.3 \mathrm{sec}$ and analysis revealed that after the settling time passes, the voltage, current and power fluctuations were stabilised. FFT was performed over the MISO converter's output waveform, and $27.3 \%$ THD was observed. Accordingly, the Kalman filter was modeled, and resultantly, THD in the output voltage was reduced to $2.1 \%$. This value is well within the allowable limit of $5 \%$, as prescribed by IEEE, for the THD of a PV system. Thus, a stable and accurate output voltage, regardless of the input variations, could be obtained from a PV based RES through the proposed scheme.

\section{ACKNOWLEDGEMENT}

The authors are thankful to the faculty of the Department of Electrical Engineering, University of Engineering and Technology, Lahore, Pakistan, for their assistance and support to accomplish the present research.

\section{CONFLICT OF INTEREST}

The authors declare no conflict of interest for the submitted article.

\section{REFERENCES}

1. T. E. C. D. Oliveira, P. M. S. Carvalho, P. F. Ribeiro, and B. D. Bonatto. PV hosting capacity dependence on harmonic voltage distortion in low-voltage grids: Model validation with experimental data. Energies 11(2): 465 (2018).

2. M. Dhananjaya, R. Pilla, and T. S. Gorripotu. Integrated multi-input DC-DC converter with reduced switches. International Transactions on Electrical Energy Systems 31(8): e12989 (2021).

3. R. P. Anggraini, M. Z. Efendi, and F. D. Murdianto. Modeling and simulation of MISO buck converter using ANFIS on DC microgrid distribution system. IOP Conference Series: Materials Science and Engineering 1096(1): (2021).

4. F. Mirzapour, M. Lakzaei, G. Varamini, M. Teimourian and N. Ghadimi. A new prediction model of battery and wind-solar output in hybrid power system. Journal of Ambient Intelligence and Humanized Computing 10(1): 77-87 (2019).

5. S. Prasad, D. Devaraj, R. Boddula, S. Salla and M. S. Alsahi. Fabrication, device performance, and MPPT for flexible dye-sensitised solar panel based on gel-polymer phthaloylchitosan based electrolyte and nanocluster $\mathrm{CoS} 2$ counter electrode. Materials Science for Energy Technologies, 2(2): 319-328 (2019).

6. M. Dhananjaya, and S. Pattnaik. Review on MultiPort DC-DC converters. IETE Technical Review 1-14: (2021).

7. R. M. Ahmed, N. E. Zakzouk, M.I. Abdelkader and A.K. Abdelsalam. Modified Partial-ShadingTolerant Multi-Input-Single-Output photovoltaic string converter IEEE Access 9: 30663-30676 (2021).

8. G. Leonides, S.P. Christopher, and L.N. Rotsios. Efficiency optimisation of MISO converter (2020). https://digitalcommons.calpoly.edu/eesp/492/ (accessed 01 August 2021).

9. N. Kacimi, S. Grouni, A. Idir and M. S. Boucherit. New improved hybrid MPPT based on neural network-model predictive control kalman filter for photovoltaic system. Indonesian Journal of Electrical Engineering and Computer Science 20(3): 1230-1241 (2020).

10. M. Ahmed, M. Abdelrahem, and R. Kennel. Highly efficient and robust grid connected photovoltaic system based model predictive control with kalman filtering capability. Sustainability 12(11): 4542 (2020).

11. S. K. Prince, K. P. Panda, and G. Panda. Kalman filter variant intelligent control for power quality improvement in photovoltaic active power filter system. International Transactions on Electrical Energy Systems 30(3): e12239 (2020).

12. G. Barchi, and D. Macii. A photovoltaics-aided interlaced extended kalman filter for distribution systems state estimation. Sustainable Energy, Grids and Networks 26: 100438 (2021). 
13. E. Farrokhi, H. Ghoreishy, R. Ahmadiahangar, and A. Rosin. Kalman-filter based maximum power point tracking for a single-stage grid-connected. photovoltaic system. IECON 2021-47th Annual Conference of the IEEE Industrial Electronics Society. (2021).

14. N. Kumari, and R. Kulkarni. Use of kalman filter and its variants in state estimation: A review. Artificial Intelligence for a Sustainable Industry 4:213-230: (2021).

15. A. Honarbari, S. N. shad, M. S. Pour, S. S. M. Ajarostaghi, and A. hassannia. MPPT improvement for PMSG-Based wind turbines using extended kalman filter and fuzzy control system. Energies 14(22): 7503 (2021).

16. S. R. Das, P. K. Ray, A. Mohanty,and G. Panda. Power quality enhancement in PV and battery storage based microgrid using hybrid active filter. $3^{\text {rd }}$ International Conference on Energy, Power and Environment: Towards Clean Energy Technologies (2021).

17. M. S. Nazar, and A. E. Fard. The adaptive NeuroFuzzy inference system model for short-term load, price, and topology forecasting of distribution system. Application of Machine Learning and Deep Learning Methods to Power System Problems 321343 (2021).

18. H. H. H. Aly, A hybrid optimised model of adaptive neuro-fuzzy interface system, recurrent kalman filter and neuro-wavelet for wind power forecasting driven by DFIG. Energy 122367 (2021).

19. S. Sathishkumar, M. V. Rajkumar, S. V. Kumar, M. Maruthamuthu, A. Soundar and A. Kumaresann. A new design for DC-DC converter topology with
MISO for renewable energy resources. International Journal of Emerging Technologies in Engineering Research (IJETER) 5(4): 143-149 (2017).

20. J. R. Stroud, M. Katzfuss, and C. K. Wikle. A bayesian adaptive ensemble kalman filter for sequential state and parameter estimation. Monthly Weather Review 146 (1): 373-386 (2018).

21. M. R. Banaei, H. Ardi, R. Alizadeh and A. Farakhor. Non-isolated multi-input-single-output DC/ DC converter for photovoltaic power generation systems. IET Power Electronics 7(11): 2806-2816 (2014).

22. P. Matisko and V. Havlena. Noise covariance estimation for Kalman filter tuning using bayesian approach and monte carlo. International Journal of Adaptive Control and Signal Processing 27(11): 957-973 (2013).

23. O. Jong. Multiple Input Single Output (MISO) DC-DC Converter for the DC House Project (2012). https://digitalcommons.calpoly.edu/cgi/ viewcontent.cgi article $=1164 \& \operatorname{context}=$ eesp (accessed 01 August 2021).

\section{DECLARATION}

Authors declare that: (i) the results are original; (ii) the same material is neither published nor under consideration elsewhere; (iii) approval of all authors have been obtained; and (iv) in case the article is accepted for publication, its copyright will be assigned to Pakistan Academy of Sciences. Authors will obtain permission to reproduce, where needed, copyrighted material from other sources and ensure that no copyrights are infringed upon. 
